\title{
Modelo de previsão hidrológica utilizando redes neurais artificiais: um estudo de caso na bacia do Rio Xingu -
}

\author{
Altamira-PA

\section{Arilson Galdino da Silva ${ }^{1}$, Adriana R. G. Castro $^{2}$ and Alen Costa Vieira ${ }^{1}$} \\ ${ }^{1}$ Centro Gestor e Operacional do Sistema de Proteção da Amazônia (CENSIPAM) Centro Regional de Belém, \\ Belém - PA - Brasil and ${ }^{2}$ Universidade Federal do Pará (UFPA) Caixa Postal 479 - 66075-110 - Belém - PA - \\ Brasil \\ *arilson.silva@sipam.gov.br;adcastro@ufpa.br;alen.vieira@sipam.gov.br
}

Submetido: 22/10/2018. Revisado: 05/11/2018. Aceito: 05/11/2018.

\begin{abstract}
Knowledge about the extent of river bed overflow is extremely necessary for the determination of areas at risk. The City of Altamira-PA, located on the banks of the Xingu River, historically suffers from extreme events of floods that provoke floods, causing great damages to the population. Considering the problem, this paper presents a monthly level prediction system of the Xingu River based on neural networks perceptron of multiple layers. For the development of the system, precipitacion data were used in the basin and sub-basins of the Xingu River, and SST information (Sea Surface Temperature) from 1979 to 2016. The satisfactory results demonstrate the great applicability of the artificial neural networks to the problem.
\end{abstract}

Key words: Hydrological Modeling; Xingu River Quota Forecasing; Neural Networks; Time Series

\section{Resumo}

O conhecimento acerca da amplitude do transbordamento dos leitos fluviais é extremamente necessário para determinação de áreas de risco. A cidade de Altamira-PA, localizada às margens do rio Xingu, vem sofrendo com casos extremos de cheias que tendem a provocar inundações, resultando em severos prejuízos para a sua população. Considerando o problema, este artigo apresenta a proposta de um sistema de previsão de nível mensal do Rio Xingu baseado em Redes Neurais Artificiais Perceptron de múltiplas camadas. Para o desenvolvimento do sistema foram utilizados dados de precipitação na bacia e sub-bacias do Rio Xingu, e informações de Temperatura da Superfície do Mar (TSM) do período de 1979 a 2016. Os resultados satisfatórios obtidos demonstram a grande aplicabilidade das Redes Neurais Artificiais para o problema de previsão de cheias.

Palavras-Chave: Modelagem Hidrológica; Previsão de Cotas do Rio Xingu; Redes Neurais, Série Temporais

\section{Introdução}

Durante o período chuvoso são frequentes notícias, tanto no âmbito regional como internacional, sobre eventos de enchentes. Como consequência, estes eventos podem ocasionar grandes prejuízos econômicos e sociais para a população atingida, tais como: interrupção da atividade econômica nas áreas inundadas; perdas materiais e humanas; contaminação por doenças de veiculação hídrica e inúmeras pessoas desabrigadas.

A cidade de Altamira-PA, localizada às margens do rio Xingu, vem sofrendo com casos extremos de cheias que tendem a provocar inundações, resultando 
em severos prejuízos para a população que reside no entorno do rio. Estes incidentes desabrigam um número grande de famílias, causando proliferação de uma série de doenças e acarretando inúmeras vulnerabilidades sociais na região (Franco; 2014; Miranda Neto; 2014; Silva et al.; 2014).

A bacia hidrográfica do rio Xingu está situada dentro dos estados do Pará e do Mato Grosso e abrange cerca de 509,7 mil km2, sendo o rio Xingu seu principal curso d'água. Esta bacia representa um dos principais contribuintes da margem direita do rio Amazonas, constituindo $60 \%$ de seu território recoberto por áreas protegidas (Villas-Boas; 2012).

As espacialidades distintas no cenário amazônico, produzidas pela sazonalidade dos rios, alternamse entre os períodos de cheias e secas. Diante destes fenômenos, existe a necessidade de realização de estudos sobre a bacia hidrográfica, tornando a determinação das áreas de risco, uma necessidade para o conhecimento acerca da amplitude do transbordamento dos leitos (Sander; 2012).

Considerando a importância do desenvolvimento de sistemas de previsão de cheias para a bacia hidrográfica do Rio Xingu, mas especificamente, para a região onde está localizada a cidade de AltamiraPA, este trabalho tem por objetivo, apresentar uma proposta de um sistema de previsão de cotas baseada em Redes Neurais Artificiais Perceptron de múltiplas camadas, com uso de informações das médias mensais das cotas do rio, séries históricas de precipitação (da bacia e sub-bacias do Rio Xingu) e dados de temperatura da superfície do mar (TSM). A escolha pelo uso de variáveis de TSM se deve aos estudos realizados em (Franco; 2014). Neste aprendizado, verificou-se que o regime hidrológico da bacia do Xingu recebe influências oceânicas do Pacífico e do Atlântico, alterando o regime de precipitação nas sub-bacias, afetando o ciclo hidrológico em Altamira, aumentando assim (ou diminuindo) o nível do rio.

As Redes Neurais Perceptron de Múltiplas camadas, vêm sendo largamente utilizadas na literatura para a solução de uma grande variedade de problemas. Com o avanço de métodos de aprendizagem e das arquiteturas, estas redes têm se mostrado ferramentas bastante promissoras, em particular, para o problema de previsão de inundações baseadas em séries temporais. Entre os trabalhos desenvolvidos na área de previsão com redes, citamse os trabalhos apresentados em (Adnan et al.; 2012; Xie et al.; 2010; Weigang et al.; 1998). Em relação a sistemas propostos para previsão de cheias em regiões no Brasil, citam-se os trabalhos de (Filippo; 2003; Dawson and Wilby; 2001; Santos; 2008; de Souza et al.; 2005).

Nas próximas seções serão descritas as redes neurais Perceptron de múltiplas camadas, a Bacia do Rio Xingu e o sistema de previsão proposto com resultados e conclusões.

\section{Redes Perceptron de Múltiplas Camadas}

Uma rede neural Perceptron multicamadas (MLP), possui um número finito de camadas sucessivas Fig. 1, cada uma com número finito de unidades de

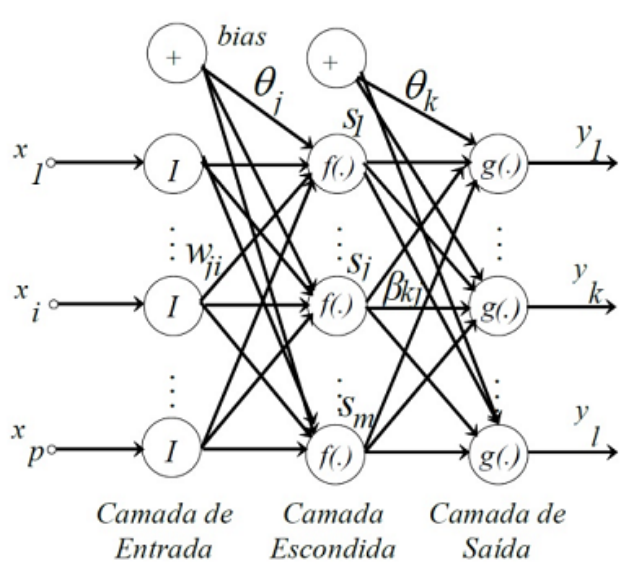

Figura 1: Rede Neural Perceptron Multicamadas

processamento, chamadas neurônios. Cada neurônio de uma camada está conectado a todos os neurônios da camada seguinte através de pesos sinápticos.

O sinal de entrada é recebido pela camada de entrada, sendo enviado posteriormente para a camada seguinte. O processo de aprendizado é realizado pelas camadas escondidas que é feito através do processamento de cada neurônio. Um neurônio de uma determinada camada, possui a função de combinar as tarefas realizadas pelos neurônios a ele ligados da camada anterior e, contribuir no processamento, auxiliando no armazenamento das informações. Uma resposta é recebida, na camada de saída, de acordo com as classes informadas no conjunto de dados, através dos vários processamentos realizados nas camadas anteriores (FACELI; 2011)

Nas redes Perceptron, MLPs, além das camadas de entrada e saída, temos a disponibilização de neurônios organizados em uma ou mais camadas ocultas, denominadas camadas intermediárias, por estarem inseridas entre a camada de entrada e saída e, sua função básica é intervir entre a camada de entrada externa e a saída da rede de maneira útil (Haykin; 2009).

Cada neurônio na camada escondida realiza o seguinte cálculo:

$$
S_{j}=f\left(\sum_{i=1}^{p} x_{i} w_{i j}+\theta_{k}\right)
$$

onde $x_{i}$ é a $i$-ésima entrada para a rede, $w_{i j}$ é o peso da conexão do neurônio de entrada $i$ para o neurônio escondido $j, \theta_{j}$ é o bias do j-ésimo neurônio e $f($.) é a função de ativação do neurônio.

Considerando a camada de saída, cada neurônio calcula:

$$
y_{k}=g\left(\sum_{j=1}^{m} \beta_{k j} S_{j}\right)+\theta_{j}
$$

onde $\beta_{k j}$ é o peso da conexão escondido $j$ com neurônio de saída $k, y_{k}$ é a $k$-ésima saída da rede, $\theta_{k}$ 


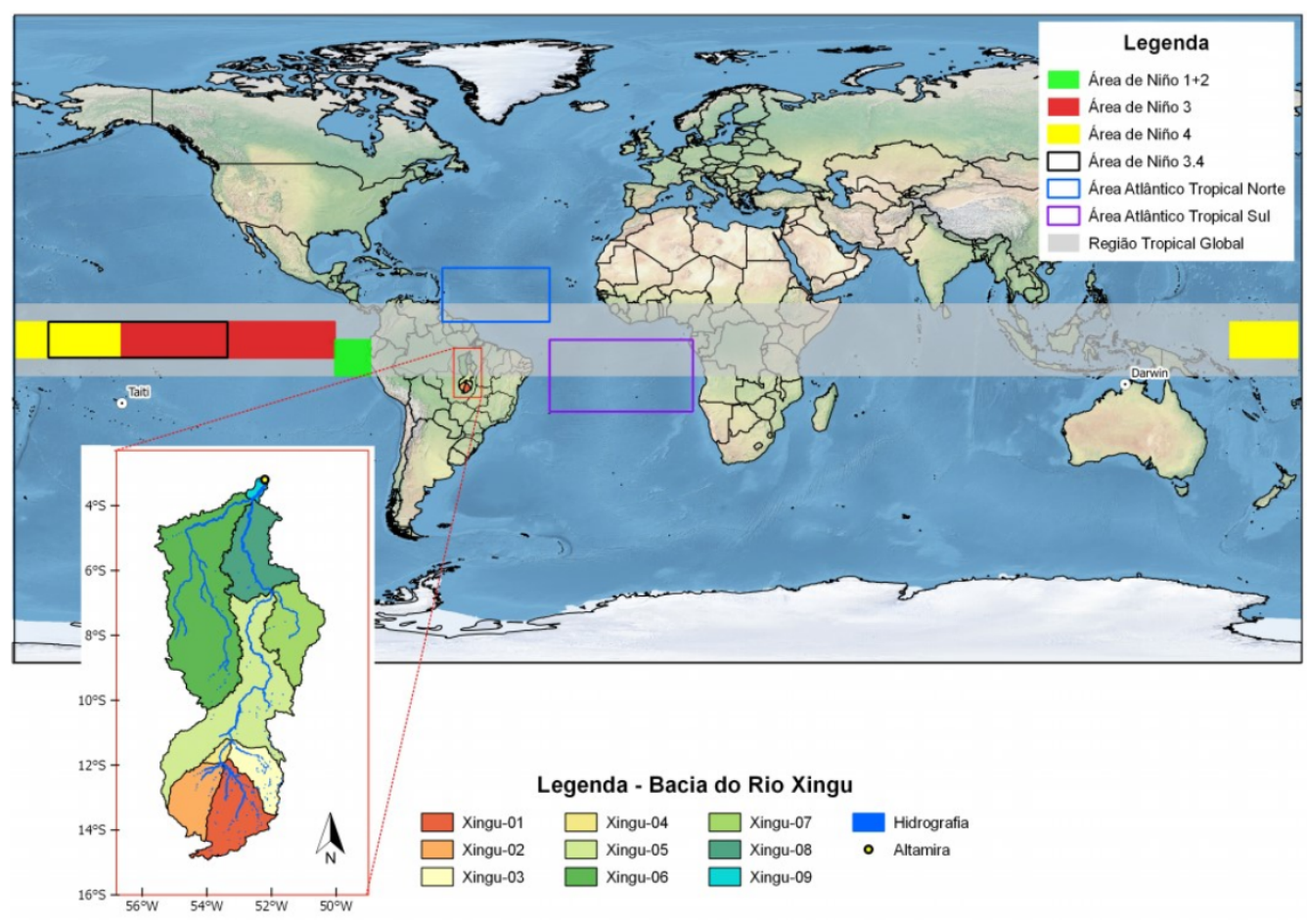

Figura 2: Mapa com áreas das 18 variáveis de interesse - Detalhamento nas sub-bacias e informações de TSM. Fonte: Sistema de Proteção da Amazônia(2016)

é o bias da $k$-ésima saída e $g($.) é a função de ativação do neurônio.

O principal objetivo do aprendizado em Redes Neurais é a obtenção de modelos com boa capacidade de generalização, tendo como base o arranjo de dados.

As MLPs, possuem a habilidade de aprender a partir de exemplos e, este aprendizado é realizado através de um processo iterativo de ajustes aplicados aos seus pesos sinápticos. Para que se possa realizar o processo de aprendizagem, é preciso primeiramente se ter um modelo do ambiente no qual a rede neural será inserida. A generalização, outra característica importante da MLP, é a capacidade de responder a situações que não foram apresentadas à rede neural na etapa de aprendizado (Castro et al.; 2011).

Através de treinamento supervisionado com algoritmo popular conhecido como algoritmo de retropropagação do erro (error backpropagation), sendo as perceptrons de múltiplas camadas bastante utilizadas para resolver problemas complexos. Assim, este algoritmo é baseado no aprendizado por correção do erro.

O processo de aprendizagem, por retropropagação do erro, é definido em dois passos, através das diferentes camadas da rede: propagação, um passo para frente e um passo para trás, a retropropagação. No processo de passo para frente, é aplicado aos nós sensoriais da rede um padrão de entrada, e seu efeito propagase em suas camadas. Como resposta, um conjunto de saídas é produzido e, no processo de propagação, ocorrem a fixação dos pesos sinápticos. Por outro lado, no passo para trás, através de uma regra de correção do erro os pesos sinápticos são ajustados. Detalhadamente, ocorre uma subtração entre a resposta real da rede e a desejada (alvo), produzindo um sinal de erro.
Segundo (Hornik; 1989), uma MLP possui a capacidade de ser uma aproximadora universal e apresentar três características específicas:

- A presença de no mínimo uma camada de neurônios escondidos, que não fazem parte da camada de entrada nem da saída da rede. Tais neurônios ocultos proporcionam a rede $o$ aprendizado de atividades complexas, retirando positivamente as informações mais importantes dos padrões de entrada da rede;

- A rede apresenta alto grau de conectividade, definido pelas sinapses da rede. Ocorre uma mudança na massa das conexões sinápticas ou de seus pesos, durante a modificação na conectividade da rede;

- Utilização de uma função de ativação não linear em cada neurônio da rede, em que essa função tenha um coeficiente de não linearidade tênue para que seja diferenciável em qualquer ponto;

A capacidade de aproximação depende, exclusivamente, da topologia da rede e da quantidade de neurônios que cada camada foi definida. Ainda assim, na literatura não temos uma conclusão a respeito do número necessário de neurônios ocultos para alcançar a ótima solução do aproximador universal.

Uma etapa de aprendizado é necessário, para a rede configurada. Assim, a rede neural torna-se vantajosa e consegue desempenhar a função para a qual foi designada. A metodologia de aprendizagem pode ser mencionada em dois paradigmas distintos: aprendizado supervisionado e não-supervisionado.

No aprendizado supervisionado, dependente do ambiente externo, ocorre a comparação da saída da rede com o valor desejado (alvo). Para minimizar o 
erro, os pesos são ajustados entre saída da rede e o valor esperado. O algoritmo de retropropagação do erro é destacado durante o processo.

Com relação ao aprendizado não-supervisionado, considerado um procedimento auto-organizável, não ocorrem comparações entre a saída da rede e o objetivo, ou seja, não depende do meio exterior. Diante do cenário configurado, a rede propriamente dita decide qual a ótima saída de acordo com determinada entrada.

O obejtivo principal do algoritmo de retropropagação é desenvolver o ajuste dos pesos sinápticos da rede neural, através da disseminação inversa do erro na camada de saída. Esse algoritmo é um método sistemático de adequação dos pesos das redes neurais apoiado no recurso do gradiente descendente (Simpson; 1999).

As redes MLPs vêm sendo largamente utilizadas para problemas de previsão de séries temporais, com muitos trabalhos desenvolvidos para área de previsão de cheias, como apresentado em (Dornelles et al.; 2013; Weigang et al.; 1998; Gopakumar and Takara; 2009; Adnan et al.; 2012; Chaowanawatee and Heednacram; 2012),(Simor et al.; 2012), Dornelles et al. (2013), (Li; 1998), (Gopakumar and Takara; 2009), Adnan et al. (2012), (Chaowanawatee and Heednacram; 2012), (Nizar Shamsuddin, Mohd Khairul et al.; 2017) e (Coutouis et al.; 2016).

A abordagem de boa capacidade de generalização, pode ser inferido quando o mapeamento da entrada com a saída da rede, em sua configuração, tiver aproximação correta, ou seja, a rede realizar uma boa interpretação diante dos dados de entrada, sendo estes dados desconhecidos pela rede, pois tais dados não foram utilizados durante a fase de treinamento (Haykin; 2009).

\section{Bacia Hidrográfica do Rio Xingu}

Ao longo do rio Xingu são contabilizados 50 municípios, sendo 35 do Estado do Mato Grosso e 15 do Estado do Pará. No Pará, ocupa uma área de aproximadamente $314.427,790 \mathrm{~km} 2$, que corresponde a $\mathbf{2 5 , 1 \%}$ do Estado. Juntamente com as bacias dos rios Tapajós e Tocantins, compõe o conjunto de bacias federais que drenam mais de $50 \%$ do território paraense. Na bacia hidrográfica do rio Xingu, destaca-se o município de Altamira, situada no sudoeste do Estado do Pará. Os rios Culuene e Sete de Setembro formam as nascentes do rio Xingu, situados em altitudes da ordem de $500 \mathrm{~m}$, na junção da Serra do Roncador coma a Serra Formosa, no Estado do Mato Grosso (IBGE; 2018). Com a subdivisão em três partes, o rio Xingu apresenta alto, médio e baixo curso. Uma zona de transição é delimitada, no trecho médio superior, pelo surgimento de terrenos em declive acentuados e com boa definição em certos pontos, intercalados com a presença, ainda predominante, de margens baixas (Brasil; 2003).

Apresentando uma topografia mais acentuada, na altura da cidade de São Félix do Xingu, perfazendo pequenas corredeiras, seguindo seu curso, são formadas ilhas e vários braços e, entre as épocas de cheia e estiagem, destaca-se sua variação de volume.

Variações climáticas são observadas de sul para o norte e que são refletidas durante o regime hidrológico. No médio curso de São Felix do Xingu no Pará, o período de chuva compreende os meses de outubro a maio, e o período de seca corresponde aos meses de junho a setembro. $O$ período chuvoso, na região de Altamira, ocorre entre dezembro e maio, e o período de seca ocorre de julho a novembro. Na região de Porto de $\mathrm{Moz}$, no baixo curso, o período de chuva está compreendido entre os meses de janeiro a julho, e o período seco entre agosto de dezembro (Eletrobrás; 2009).

A caracteristica de grande volume de água e apresentação de relevo acidentado, proporciona a esta região um alto potencial hidrelétrico, denotando estruturas ideais para a geração de energia. Diante deste potencial, depois de anos de estudos e debates, está sendo instalado o Aproveitamento Hidrelétrico, chamado AHE Belo Monte com potência instalada de 233,1 MW, composta de nove turbinas, do modelo bulbo, com potência unitária de 25,9 MW, estabelecida a $40 \mathrm{~km}$ rio abaixo, após a cidade de Altamira. O projeto define o desvio de grande parte do curso de água do rio Xingu, em um trecho de mais ou menos 100 quilômetros, que é denominada como Volta Grande do Rio Xingu. Sendo que esta sofrerá uma rigorosa redução dos níveis de água no trecho amputado do rio (Berman; 2012).

\section{Sistema de Previsão de Cotas Proposto}

\subsection{Base de Dados para Desenvolvimento do Sistema}

Para o desenvolvimento do sistema de previsão de cotas proposto, foram utilizados dados de níveis médios mensais do rio Xingu na estação de Altamira, os dados de médias mensais de temperatura e pressão dos oceanos Atlântico e Pacífico, e os dados de estimativas de precipitação nas sub-bacias do Rio Xingu para o período de 1979 até 2016.

Os dados de níveis médios mensais do rio Xingu foram obtidos no Banco de Dados Hidrometeorológico da Agência Nacional de Águas ANA (2017), sendo a rede hidrometeorológica observacional operada na Amazônia através da Companhia de Pesquisa em Recursos Minerais (CPRM).

Os dados de médias mensais de temperatura e pressão dos oceanos Atlântico e Pacífico, e os dados de estimativas de precipitação, são oriundos de observações de satélite e interpolados com dados de estações pela National Oceanic and Atmosferic Administration (NOAA).

Para o cenário de previsão, conforme Fig. 2, foram utilizadas informações de precipitação e variáveis oceânicas e detalhamento das sub-bacias do rio Xingu. As séries temporais para a identificação de níveis mensais do rio Xingu em Altamira são:

- Dados de temperatura da superfície do mar (TSM) para os oceanos Atlântico norte, Atlântico sul e Atlântico tropical;

- Índices de niño (niño1+2, niño3, niño4 e niño3+4);

- Dados de pressão Taiti e Darwin;

- Informações de precipitação das 9 sub-bacias do Xingu; 


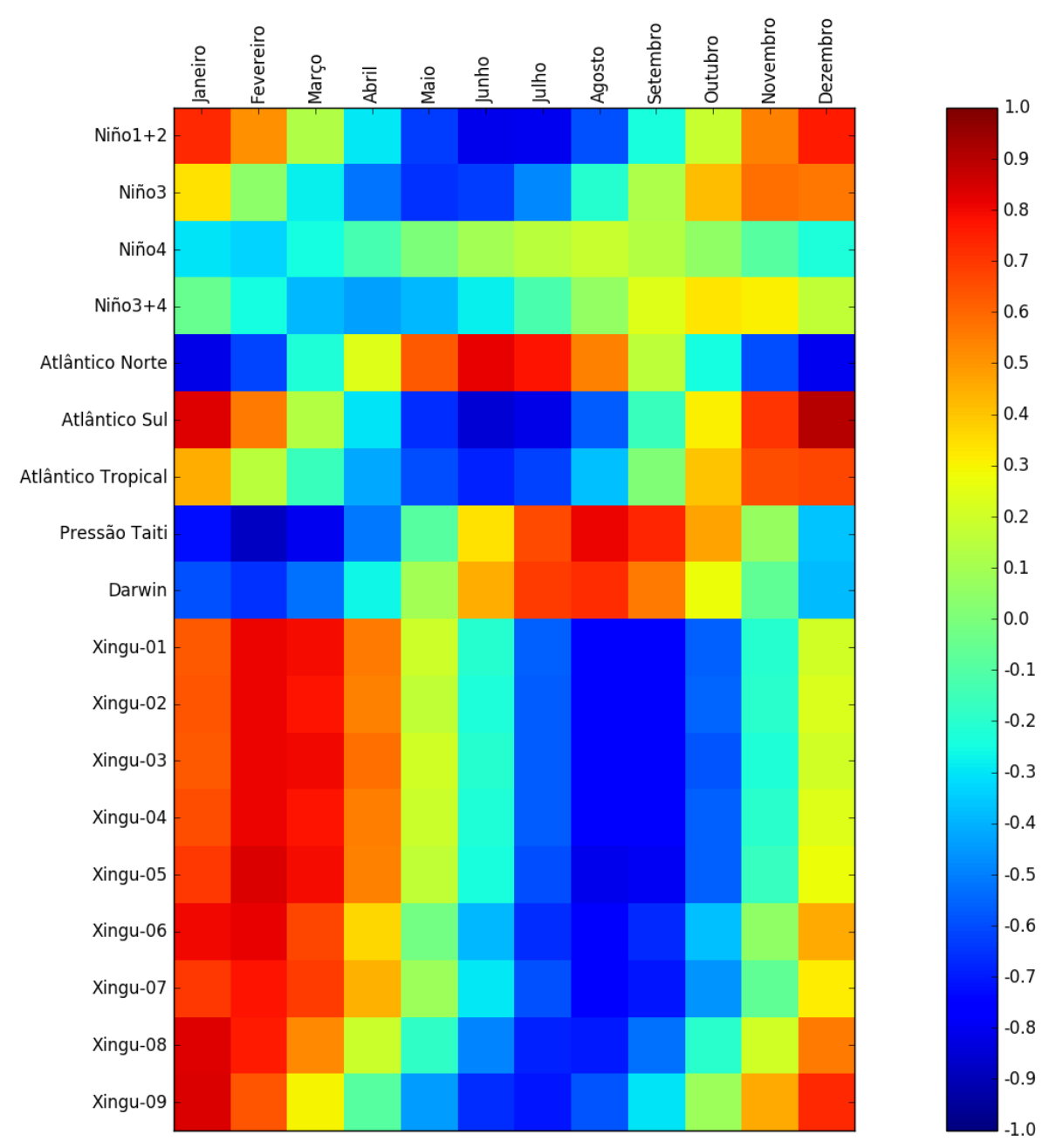

Figura 3: Correlações das 18 variáveis de interesse com o nível do rio em diferentes defasagens.

Assim, foram totalizadas 9 variáveis oceânicas (TSM) e 9 variáveis de precipitação.

Para calcular a janela de tempo que uma variável leva para afetar o nível do rio Xingu, na cidade de Altamira-PA, utilizou-se o método de correlação linear simples. Para a escolha das variáveis de entrada para o sistema de previsão, optou-se por variáveis com correlação forte, ou seja, com valores acima de 0.7 (positiva ou negativa).

Após a análise de correlação entre as variáveis de TSM (e suas defasagens no tempo) e a cota (nível) de Altamira, as variáveis selecionadas com correlação acima de 0,7 foram: Variável El-niño1+2 com defasagens de 1,6,7 e 12 meses; Variável Atlântico norte com defasagens de 1,6,7 e 12 meses; Variável Atlântico sul com defasagens de 1,6,7,11 e 12 meses; Variável Pressão Darwin com defasagens de 1,2,3,8 e 9 meses; e Variável Pressão Taiti com defasagem de 8 meses;

Realizado estudos da correlação com as variáveis de precipitação das sub-bacias do rio Xingu (e suas defasagens) e a cota (nível) de Altamira. As variáveis selecionadas com correlação acima de 0,7 foram: Variável sub-bacia Xingu-01 com defasagens de 2,3,8 e 9 meses; Variável sub-bacia Xingu-02 com defasagens de 2,3,8 e 9 meses; Variável subbacia Xingu-03 com defasagens de 2,3,8 e 9 meses; Variável sub-bacia Xingu-04 com defasagens de
2,3,8 e 9 meses e; Variável sub-bacia Xingu-05 com defasagens de 2,3,8 e 9 meses; Variável sub-bacia Xingu-06 com defasagens de 1,2, e 8 meses; Variável sub-bacia Xingu-07 com defasagens de 2,8 e 9 meses; Variável subbacia Xingu-08 com defasagens de 1 e 2 meses e Variável sub-bacia Xingu-09 com defasagens de 1,7 e 12 meses.

Conforme a Fig. 3, observa-se as variáveis, TSM ou precipitação, que possuem maior influência para a previsão do sistema. Assim, temos uma base de informações mais limpa e customizável, no que diz respeito aos dados que realmente conseguem contribuir para o resultado desejado.

A seleção das variáveis que serão utilizadas durante a análise de previsão, podem constituir fator determinante para composição dos resultados. A uso da abordagem de correlação visa buscar entre quais dados serão melhor disponibilizados para se alcançar o objetivo.

Através da abordagem de correlação, algumas variáveis desempenham papel importante na modelagem e previsão, já em outros cenários possuem uma contribuição mínima, podendo até serem descartadas, para não incorrer em informações inutilizáveis, ocasionando uma sobrecarga durante o processamento de previsão. 
Tabela 1: Quadro comparativo de treinamento da MLP - Entrada com dados de TSM (Temperatura da superfície do Mar)

\begin{tabular}{ccccc}
\hline Número de neurônios na camada escondida: & MSE Treino & MSE Validação & MSE Teste & $R^{2}$ \\
\hline 10 Neurônios & 0.00158 & 00.00103 & 0.00233 & 0.9923 \\
\hline 20 Neurônios & $9.33 \times 10^{-4}$ & $9.21 \times 10^{-4}$ & $4.83 \times 10^{-4}$ & 0.9957 \\
\hline 25 Neurônios & $.231 \times 10^{-4}$ & $1.78 \times 10^{-4}$ & $3.93 \times 10^{-5}$ & 0.9990 \\
\hline 30 Neurônios & $2.33 \times 10^{-4}$ & $1.72 \times 10^{-4}$ & $3.08 \times 10^{-4}$ & 0.9989 \\
\hline 35 Neurônios & $5.49 \times 10^{-4}$ & $7.67 \times 10^{-4}$ & $9.51 \times 10^{-4}$ & 0.9958 \\
\hline 40 Neurônios & $2.04 \times 10^{-4}$ & $1.55 \times 10^{-4}$ & $2.99 \times 10^{-4}$ & 0.9991 \\
\hline
\end{tabular}

Tabela 2: Quadro comparativo de treinamento da MLP - Entrada com dados de Precipitação

\begin{tabular}{ccccc}
\hline Número de neurônios na camada escondida: & MSE Treino & MSE Validação & MSE Teste & $R^{2}$ \\
\hline 10 Neurônios & 0.05083 & 0.06415 & 0.07326 & 0.6059 \\
\hline 20 Neurônios & 0.06101 & 0.11137 & 0.11563 & 4359 \\
\hline 25 Neurônios & 0.03684 & 0.07755 & 0.8510 & 0.6703 \\
\hline 30 Neurônios & 0.03324 & 0.05804 & 0.08855 & 0.6552 \\
\hline 40 Neurônios & 0.00569 & 0.11444 & 0.11190 & 0.7521 \\
\hline 50 Neurônios & $3.94 \times 10^{-4}$ & 0.14655 & 0.09318 & 0.7536
\end{tabular}

Tabela 3: Quadro comparativo de treinamento da MLP - Entrada com dados de TSM e Precipitação

\begin{tabular}{ccccc}
\hline Número de neurônios na camada escondida: & MSE Treino & MSE Validação & MSE Teste & $R^{2}$ \\
\hline 10 Neurônios & $4.92 \times 10^{-4}$ & 0.00775 & 0.1196 & 0.9711 \\
\hline 15 Neurônios & $7.35 \times 10^{-4}$ & 0.00617 & 0.01033 & 0.9733 \\
\hline 20 Neurônios & 0.00467 & 0.00762 & 0.02264 & 0.9474 \\
\hline 25 Neurônios & 0.00133 & 0.00817 & 0.01219 & 0.9644 \\
\hline 30 Neurônios & $1.26 \times 10^{-4}$ & 0.00631 & 0.01105 & 0.9781 \\
\hline 35 Neurônios & $9.78 \times 10^{-4}$ & 0.00731 & 0.02210 & 0.9661 \\
\hline
\end{tabular}

\section{Resultados}

Para desenvolvimento do sistema de previsão de cotas, utilizou-se uma rede neural MLP. Para treinamento da rede foram utilizadas informações do período de 1979 a 2016, separados em $70 \%$ dos dados para treinamento da rede, $15 \%$ para validação e $15 \%$ para testes.

A estratégia de alocação da quantidade de neurônios, para o sistema proposto, deve-se aos resultados obtidos diante da configuração apresentada e, devido o aumento da quantidade de neurônios, não foi possível obter melhores resultados.

De acordo com (Dawson and Wilby; 2001) a avaliação de precisão em cada treinamento foi realizada utilizando o coeficiente de determinação $\left(R^{2}\right)$ e o erro médio quadrático (MSE).

O coeficiente de determinação permite medir a qualidade do sistema de previsão em relação à sua capacidade de prever corretamente os valores da resposta, podendo ser medido através da equação:

$$
R^{2}=1-\frac{\sum_{i=1}^{n}\left(\hat{y}_{i}-y_{i}\right)^{2}}{\sum_{i=1}^{n}\left(y_{i}-\bar{y}\right)^{2}}
$$

Onde $y_{i}$ é o valor observado, $\hat{y}_{i}$ é o valor estimado (previsão) e $\bar{y}$ é a medida das observações.

Quanto mais próximo de 1 estiver o $R^{2}$, muito melhor será o desempenho do modelo. O Erro Quadrático Médio (MSE) pode ser determinado através de:

$$
M S E=\frac{1}{n} \sum_{i=1}^{n}\left(\hat{y}_{i}-y_{i}\right)^{2}
$$

onde $\hat{y}_{i}$ é o valor estimado (previsão) e $y_{i}$ é o valor observado de série histórica.

Foram realizados três experimentos com as seguintes variáveis para o sistema de previsão de cotas do rio (previsão para o mês seguinte):

- Experimento 1: Dados de entrada da rede neural, com informações apenas de TSM, sendo utilizadas as 19 entradas selecionadas após a análise de correlação;

- Experimento 2: Dados de entrada da rede neural, com informações apenas de Precipitação, sendo utilizadas as 31 entradas selecionadas após a análise de correlação;

- Experimento 3: Dados de entrada da rede neural, com informações de Precipitação em conjunto com dados de TSM, sendo utilizadas as 50 entradas após a análise de correlação.

Para os Experimentos 1, 2 e 3, diversos treinamentos da MLP foram realizados variando-se o número de neurônios na camada escondida. As Tabelas 1, 2 e 3 apresentam alguns dos melhores resultados obtidos, respectivamente.

Conforme resultados obtidos, para o Experimento 1, a topologia que apresentou melhores resultados foi a topologia com 40 neurônios na camada intermediária. Para o Experimento 2, o melhor resultado foi obtido com a topologia contendo 50 neurônios na camada escondida. Para o Experimento 3, a topologia com 30 


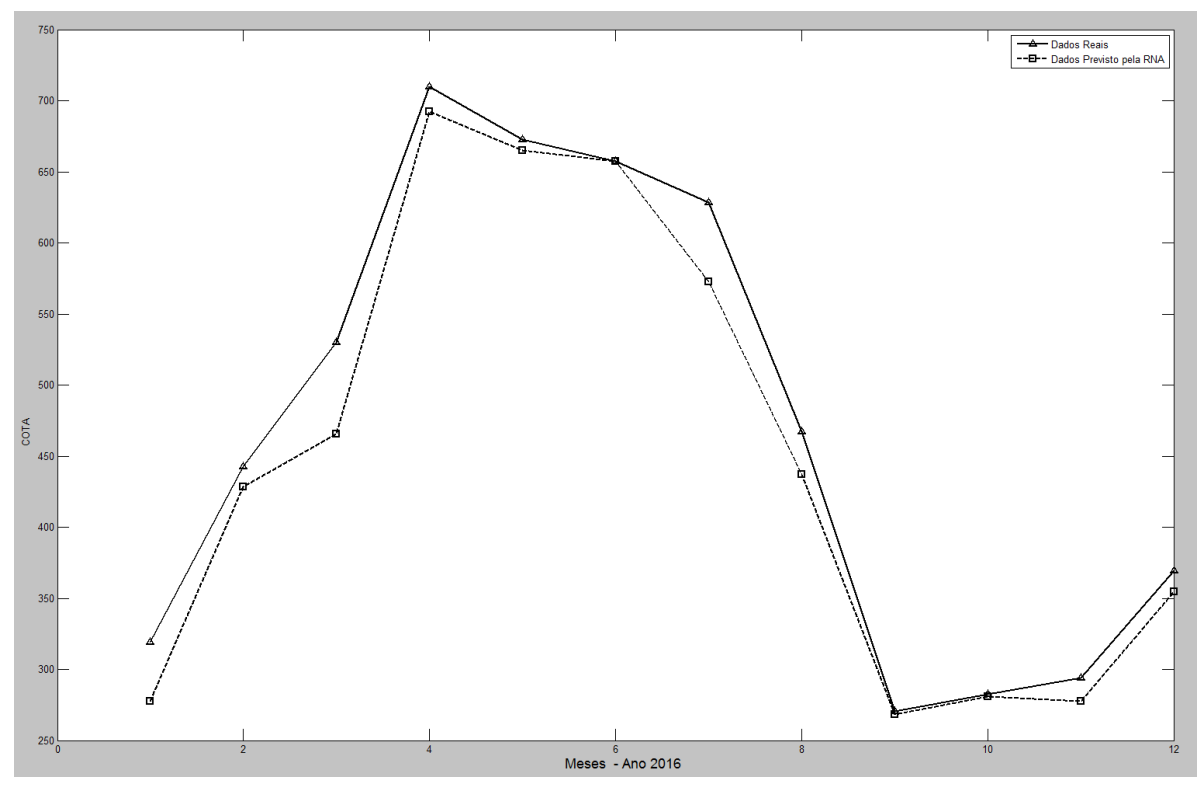

Figura 4: Previsão de cota para o ano de 2016.

neurônios na camada escondida apresentou melhor desempenho.

Algumas topologias, com mais de 50 neurônios, foram configuradas e testadas, no entanto não apresentaram resultados satisfatórios, diante do mapeamento realizado com os experimentos em tela. Nesse sentido, denota-se a capacidade de generalização da rede neural em sintetizar os resultados obtidos.

O melhor resultado, dentre todos os testes realizados, foi obtido quando se utilizou somente informações de TSM (Temperatura da superfície do mar), como dado de entrada da rede neural, obtendo valor de R $2=0.9991$. Conforme Fig. 4, são demonstrados resultados de previsão para $\mathbf{0}$ ano de 2016 para a topologia com 30 neurônios.

Os resultados satisfatórios com relação ao uso de dados de TSM, denotam a grande utilidade das redes neurais, no processo de tomada de decisão, no que diz respeito a quais variáveis utilizar durante os procedimentos de previsão de cotas para determinada região.

Em (Franco; 2014; Miranda Neto; 2014; Silva et al.; 2014), para dados de mesma origem usados neste trabalho, foram obtidos valores de R $2=0.89$ com a utilização do método de regressão linear e regressão múltipla. Através do resultado superior obtido com o sistema proposto, demonstra-se assim a aplicabilidade das redes perceptron para o problema de previsão de cotas do rio Xingu.

\section{Conclusão}

A previsão de eventos hidrológicos extremos, principalmente na região Amazônica, pode auxiliar na resolução de problemas de cheias, evitando perdas humanas e materiais em detrimento de uma antecipação de informação. Este trabalho apresentou uma proposta de sistema de previsão de cotas do rio Xingu, na cidade de Altamira-PA, usando redes MLP.

A sistematização para previsão de cheias evoluiram bastante nos últimos anos, porém conforme observado a utilização de técnicas tradicionais, operada pelas entidades ou sistemas de apoio, geralmente são adequadas para aplicação em condições normais, entretanto podem não predizer um resultado, de forma satisfatória, em situações onde ocorram mudanças inesperadas nas variáveis de ambiente. Complexos relacionamentos extremos entre as variáveis podem levar a execuções matemáticas obscuras, no momento da previsão, tornado um fator negativo durante a análise.

Para o desenvolvimento do sistema foram realizados estudos da correlação, entre possíveis variáveis de entrada para o sistema proposto. O bom resultado obtido demonstra a grande aplicabilidade das redes perceptron para o problema em questão, sendo importante destacar que o uso de informações de temperatura da superfície do mar, propiciou modelos de previsão mais eficazes, mostrando desta forma o quanto estas informações podem influenciar diretamente no nível do rio Xingu.

Como trabalhos futuros propõe-se a utilização da técnica com RNA para previsão em conjunto com Otimização por Enxame de Partículas (PSO), Algoritmo Genético ou Máquinas de Vetores de Suporte.

\section{Referências}

Adnan, R., Ruslan, F. A., Samad, A. M. and Zain, Z. M. (2012). Artificial neural network modelling and flood water level prediction using extended kalman filter, 2012 IEEE International Conference on Control System, Computing and Engineering, pp. 535-538.

ANA (2017). Agência Nacional de Águas. Hidro Web Sistema de Informações hidrológicas. Disponível em: http://hidroweb.ana.gov.br (Acesso em: 20 Junho 2017).

Berman, C. (2012). O projeto da Usina Hidrelétrica Belo Monte: a autocracia energética como paradigma, Vol. 15, Novos cadernos NAEA. 
Brasil (2003). Ministério do Meio Ambiente. Estudos Preliminares e Formulação de uma Proposta Técnica para Implantação de um Mosaíco de Unidades de Conservação no Médio Xingu, Brasília. MMA-ISA.

Castro, A. R. G., Miranda, V. and Lima, S. (2011). Transformer fault diagnosis based on autoassociative neural networks, 2011 16th International Conference on Intelligent System Applications to Power Systems, pp. 1-5.

Chaowanawatee, K. and Heednacram, A. (2012). Implementation of cuckoo search in rbf neural network for flood forecasting, 2012 Fourth International Conference on Computational Intelligence, Communication Systems and Networks, pp. 22-26.

Coutouis, A., Johannet, A., Ayral, P. and Cadilhac, L. (2016). Towards a neural networks-based prediction tool devoted low water-levels forecasting: relevant architecture selection based on main physical process, 8th International Congress on Environmental - Modelling and Software .

Dawson, C. W. and Wilby, R. L. (2001). Hydrological modelling using artificial neural networks, Progress in Physical Geography: Earth and Environment 25(1): 80-108.

de Souza, E. B., Kayano, M. T. and Ambrizzi, T. (2005). Intraseasonal and submonthly variability over the eastern amazon and northeast brazil during the autumn rainy season, Theoretical and Applied Climatology 81(3): 177-191.

Dornelles, F., Goldenfum, J. A. and Pedrollo, O. C. (2013). Artificial neural network methods applied to forecasting river levels, Revista Brasileira de Recursos Hídricos 18(4): 45-54.

Eletrobrás (2009). AAI - Avaliação Ambiental Integrada. Aproveitamentos hidrelétricos da Bacia Hidrográfica do Rio Xingu.

FACELI, Katti; LORENA, A. C. G. J. C. A. (2011). Inteligência Artificial: uma abordagem de aprendizagem de máquina, LTC, Rio de Janeiro.

Filippo, A. M. (2003). Variabilidade do Nível do Mar em Função de Eventos Meteorológicos de Baixa Freqüência, Doutorado em geoquímica ambiental, Universidade Federal Fluminense, Niterói, Brasil.

Franco, V. d. S. (2014). Previsão Hidrológica de cheia sazonal na sub-bacia do rio Xingu e análise de vulnerabilidade social em Altamira-PA, Mestrado em ciências ambientais, Universidade Federal do Pará, Porto Alegre, Brasil.

Gopakumar, R. and Takara, K. (2009). Water level forecasting in the vembanad water system using artificial neural networks, 2009 International Conference on Advances in Recent Technologies in Communication and Computing, pp. 948-953.

Haykin, S. S. (2009). Neural networks and learning machines, third edn, Pearson Education, Upper Saddle River, NJ.

Hornik, K; Stimchcombe, M. W. H. (1989). Multilayer feedforward networks are Universal Approximators, Vol. 2, Neural Network.
IBGE (2018). Instituto Brasileiro de Geografia e Estatística. Disponível em: https://mapas .ibge.gov.br (Acesso em: 10 Março 2018).

Li, W.; Sá, L. A. G. G. P. B. R. M. (1998). Prediction of the level of paraguay river using neural networks, 33(Especial): 1791-1797.

Miranda Neto, J. Q. d. (2014). Reassentamento da população urbana diretamente afetada pelo empreendimento hidrelétrico de belo monte em altamira-pa, Revista Nacional de Gerenciamento de Cidades.

Nizar Shamsuddin, Mohd Khairul, Mohd Kusin, Faradiella, Sulaiman, Wan Nor Azmin, Ramli, Mohammad Firuz, Tajul Baharuddin, Mohamad Faizal and Adnan, Mohd Shalahuddin (2017). Forecasting of groundwater level using artificial neural network by incorporating river recharge and river bank infiltration, MATEC Web Conf. 103: 04007. URL: https://doi.org/10.1051/matecconf/201710304007

Sander, C.; Evangelista, R. A. O. W. F. L. M. C. E. M. (2012). Cheias do rio branco e eventos de inundação na cidade de boa vista, roraima., Acta Geográfica 6(12): 41-57.

Santos, D. M. (2008). Análise e modelagem hidrometeorológica na Bacia do Rio Tocantins em Marabá-PA, Mestrado em ciências ambientais, Universidade Federal do Pará, Belém, Brasil.

Silva, C. A., Moreira, R. P. and Herrera, J. A. (2014). Usina hidrelétrica de belo monte: o processo de des-reterritorialização na (i)mobilidade em altamira/pa., Anais do VII Congresso Brasileiro de. Geógrafos, Vitória: UFES/AGB, 2014.

Simor, V., Hlavcová, K., Kohnová, s. and Szolgay, J. (2012). Application of artificial neural networks for etimating index floods, 42(4): 295-311.

Simpson, P. K. (1999). Artificial neural systems: Foundations, paradigms, applications, and implementations, Pergamon Press.

Villas-Boas, A. (2012). De olho na bacia do Xingu, Instituto Socioambiental (ISA), São Paulo.

Weigang, L., Sá, L. D. d. A., Galvão, G. P. and Bevilaqua, R. M. (1998). Prediction of the level of paraguay river using neural networks., Pesquisa Agropecuaria Brasileira .

Xie, J., Wang, T., Zhang, J. and Shen, Y. (2010). A method of flood forecasting of chaotic radial basis function neural network, 2010 2nd International Workshop on Intelligent Systems and Applications, pp. 1-5. 